\title{
The Pulsating White Dwarf L19-2
}

\author{
Denis J. Sullivan \\ Department of Physics, Victoria University of Wellington, NZ
}

\begin{abstract}
Previous studies of the southern DAV white dwarf L19-2 have revealed that it pulsates non-radially in at least five modes with periods $\sim 10^{2} \mathrm{~s}$ and amplitudes less than $10 \mathrm{mmag}$. All of these modes showed fine structure splitting, but the one-day aliases resulting from the gaps in the single-site data made identification of the various frequencies, at best, difficult. Recent observations of L19-2 obtained from Mt. John (NZ) are presented, along with a discussion of the prospects for a Whole Earth Telescope run on this object scheduled for 1995.
\end{abstract}

\section{Introduction}

A significant number of degenerate stars are observed to pulsate, and it appears likely that most, if not all, of these objects will develop instabilities leading to pulsation as they slowly cool and evolve. The degenerate pulsators appear to be grouped into several distinct classes: the very hot DOV ( $\mathrm{T}_{e} \sim 10^{5}$ degrees) and PNNV objects; the relatively hot helium atmosphere DBV stars $\left(\mathrm{T}_{e} \sim 22,000^{\circ}\right)$; and the cooler hydrogen atmosphere DAV white dwarfs $\left(\mathrm{T}_{e} \sim 12,000^{\circ}\right)$.

All of these pulsators show periods between $\sim 10^{2} \mathrm{~s}$ and $10^{3} \mathrm{~s}$, arising from nonradial $g$-mode pulsations of the fluid sphere. The DOV and DBV pulsators, in particular, display such a rich array of frequencies, that Whole Earth Telescope (WET) multi-site observations have proven to be essential in the identification process (e.g., Winget et al. 1991, 1994). A comparison of the observational data with numerical models has been so fruitful, that the descriptive term 'asteroseismology' has increasingly been used.

Some of the DAV pulsators display a much simpler $g$-mode spectrum. L19-2 is one of these. However, in some cases the modes are closely spaced in frequency (as a result of rotational or magnetic degeneracy removal), and then extended (multi-site) data sets are also required to overcome frequency aliasing problems in the data interpretation.

Interestingly, the pulsation modes of the cool white dwarfs are remarkably stable: the amplitudes may be variable, but the pulsation frequencies remain constant (as a direct result of the rigid geometrical constraints of the nearly static white dwarf structure). However, pulsation theory for these objects does predict very small period changes, resulting from evolutionary cooling effects. The pre-white dwarf DOV star, PG1159, has revealed a definite period change (Winget et al. 1991), but only one cooler DA object has demonstrated definite indications of a change (Kepler et al. 1991). 
O'Donoghue \& Warner (OW) (1987) undertook extensive observations of L19-2 between 1979 and 1985 and were able to infer the presence of five coherent pulsations with periods near $350,192,143,118$ and $113 \mathrm{~s}$, and corresponding amplitudes of $1.0,6.0,0.55,1.8$ and $2.2 \times 10^{-3} \mathrm{mag}$, respectively. Using their combined data set, OW discussed mode identification, rotational frequency splitting effects (including an inferred second-order effect), and the stability of the principal frequency.

\section{MJUO observations}

Commencing in 1994, a programme of regular high-speed photometry of L19-2 has been carried out in New Zealand at Mount John University Observatory (MJUO). A two-star photometer attached to the one metre telescope is used to obtain high-speed "white" light photometry. The essential aim of the project is to provide a supporting data base for a WET run on this object. MJUO, with a latitude of $-44^{\circ}$, is an ideal place to observe an object as far south as $-81^{\circ}$ declination throughout the year.

The MJUO data clearly shows the dominant $192 \mathrm{~s}$ oscillation detected in the previous work, as well as the $118 \mathrm{~s}$ and $113 \mathrm{~s}$ oscillations. A detailed inspection of the discrete Fourier transform (DFT) spectrum of a three-night photometric data set $(5,7$ and $8 \mathrm{Dec}$ ) reveals excess power (relative to the DFT window function) in the vicinity of these 3 obvious components. This is undoubtedly the fine structure splitting detected by OW.

\section{A WET campaign on L19-2}

L19-2 has been included as one of the objects for the first semester 1995 WET campaign. A quality (extended coverage) WET data set (even though the object will be inaccessible to northern hemisphere observers) should improve the DFT spectral window function such that the various frequency components in the light curve are unambiguously identified. Inspite of the comprehensive nature of the $\mathrm{OW}$ data set, aliasing problems resulting from the single-site data gaps made some of their conclusions arguable (including some frequency identifications and the apparent second-order rotational splitting effect).

The OW analysis led to an upper limit on the period change of the 192s pulsation of $3 \times 10^{-14} \mathrm{ss}^{-1}$. Given the relative dominance of this mode, and the fact that L19-2 is one of the hotter DAV objects, it is a good candidate to continue searching for secular cooling effects in these white dwarfs. A WET data set on this object will contribute significantly to this programme.

\section{References}

Kepler, O., et al. 1991, ApJ, 378, L48

O'Donoghue, D., \& Warner, B. 1987, MNRAS, 228, 949

Winget, D.E., et al. 1991, ApJ, 378, 326

Winget, D.E., et al. 1994, ApJ, 430, 839 